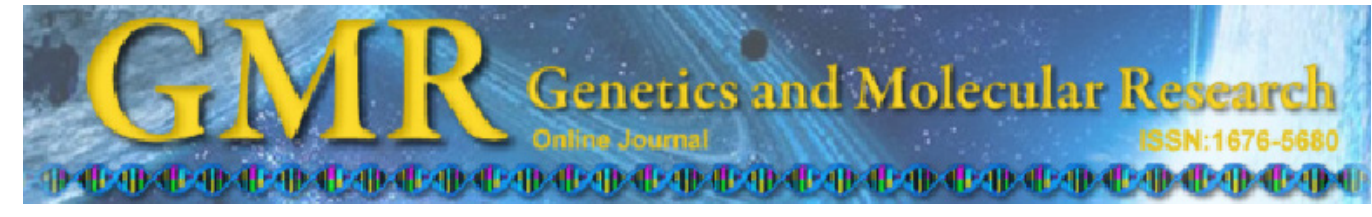

\title{
KLK1 A1789G gene polymorphism and the risk of coronary artery stenosis in the Chinese population
}

\author{
Q.D. Li*, F.J. Li*, X.C. Liu and H. Jiang \\ Key Laboratory of Cardiovascular Remodeling and Function Research, \\ Chinese Ministry of Education and Chinese Ministry of Health, \\ Qilu Hospital, Shandong University, Jinan, Shandong, China \\ *These authors contributed equally to this study. \\ Corresponding author: Q.D. Li \\ E-mail: daqingli611@163.com
}

Genet. Mol. Res. 12 (2): 1636-1645 (2013)

Received June 28, 2012

Accepted November 19, 2012

Published May 14, 2013

DOI http://dx.doi.org/10.4238/2013.May.14.4

\begin{abstract}
Previous reports have shown that polymorphism of the human tissue kallikrein 1 (KLK1) A1789G gene is associated with susceptibility to hypertension. The current study aimed to confirm the association between the polymorphism in $K L K 1$ and coronary artery stenosis (CAS). A total of 458 patients with CAS and 482 controls were used in a case-control study carried out between January 2008 and January 2011 at the Qilu Hospital (Jinan, China). Analyses of the KLK1 A1789G genotype were performed, and the logistic regression model was used to assess the odds ratio related to CAS. The results showed that the frequencies of the $\mathrm{AA}, \mathrm{AG}$, and $\mathrm{GG}$ genotypes were $11.4,50.2$, and $38.4 \%$, respectively, in patients with CAS, and $21.2,47.7$, and $31.1 \%$, respectively, in controls. Compared with the AA genotype, the GG and AG/GG genotypes were associated with a significantly increased risk of CAS. Furthermore, the AG and GG genotypes combined with smoking showed a remarkable increase in the risk for CAS. In conclusion, polymorphism of the KLK1 A1789G
\end{abstract}


gene is associated with CAS, and smoking combined with the KLK1 GG genotype was significantly associated with an increased risk of CAS. This information is extremely important to prevention strategies for CAS.

Key words: KLK1 A1789G gene; Association risk; Polymorphism; Coronary artery stenosis

\section{INTRODUCTION}

The risk factors for coronary artery disease (CAD) can be classified into 2 main categories: modifiable and non-modifiable. Modifiable risk factors include cigarette smoking, hypertension, diabetes mellitus, elevated total cholesterol, low-density lipoprotein cholesterol (LDL-C), plasma triglycerides (TGs), reduced high-density lipoprotein cholesterol (HDL-C), left ventricular hypertrophy, thrombogenic risk factors with or without medication, physical inactivity, psychological factors, elevated lipoprotein (type a), elevated homocysteine, alcohol consumption, oxidative stress, and body mass index, whereas non-modifiable risk factors include age, male gender, low socioeconomic status, and family history of premature CAD (Yeolekar and Jadhav, 1993).

The human tissue kallikrein 1 (KLK1) gene is located on chromosome 19 (19q13.2q13.4) together with several homologous genes coding for non-kinin-forming serine proteases or unidentified protein products. The $K L K 1$ gene spans $5.2 \mathrm{~kb}$ containing 5 exons and codes for an inactive prokallikrein form activated by the intracellular proteolysis of a short amino-terminal peptide (Slim et al., 2002). Animal studies have shown that the KLK1 gene is involved in bradykinin coronary outflow (Koch et al., 2006) and carotid artery neointima formation after balloon angioplasty (Yu et al., 2010).

The A1789G (rs5517) polymorphism of the KLK1 gene exon 4 is a single-nucleotide substitution that causes the amino acid substitution Lys $\rightarrow$ Glu. The genetic variant may influence urinary kallikrein activity and plasma creatinine $(\mathrm{Cr})$ levels in Chinese subjects with hypertension (Pravenec et al., 1991). Recently, a significant association between the KLK1 A1789G polymorphism and hypertension was found in a large northern Han Chinese population (Berry et al., 1989; Hong et al., 2005). Hypertension is closely linked to cardiovascular disease risk factors. Coronary artery stenosis (CAS) is a major part of CAD. Given these reports, polymorphism of the KLK1 A1789G gene may be associated with susceptibility to CAD. However, to the best of our knowledge, this association has not been confirmed in published reports. In the present study, we tested the association between the KLK1 A1789G polymorphism and CAS.

\section{MATERIAL AND METHODS}

\section{Subjects}

All subjects were consecutively recruited from January 2008 until January 2011 in the Qilu Hospital of Shandong University, Jinan, China. A total of 458 patients with CAS were enrolled in the study. CAS was diagnosed according to the World Health Organization 
definition and based on coronary artery angiography. The exclusion criteria included clinical evidence of acute inflammation, tumor, rheumatic condition checked by elevated C-reactive protein and erythrocyte sedimentation rate, and acute renal failure. Coronary angiograms were evaluated by experienced authors blinded to the study plan to assess atherosclerotic involvement. Stenosis score was calculated, defined as the most severe score of each vessel graded 1 for $1-25 \%$ diameter stenosis, 2 for $25-50 \%$ diameter stenosis, 3 for $50-75 \%$ diameter stenosis, and 4 for more than $75 \%$ diameter stenosis. The total coronary arterial tree was divided into 4 segments that included the left main artery, left anterior descending branch of the left main coronary artery, circumflex branch of the left main coronary artery, and right coronary artery.

The control subjects $(\mathrm{N}=482)$ were selected based on the following criteria: matched by gender and hospital, orthopedic trauma patients without clinical symptoms of CAD, and no abnormal findings on electrocardiogram (ECG) or cardiac ultrasound examinations. A medical and family history and other general information, such as smoking and drinking habits, were obtained for each subject. The research protocol was approved by the Ethics Committee of Shandong University.

Sample size was calculated using the Power 3.0 software (http://dceg.cancer.gov/bb/ tools/power). We recalculated the sample size based on the following parameters: design: case-control study including 2 exposure factors; case: control $=1: 1$; probability of CAS $=$ 0.001 for subjects aged 40-79 years at baseline; one exposure factor was genotype of $K L K 1$ A1789G, which observed a minor allele frequency of 0.20 ; another exposure factor was smoking or hypertension/diabetes; a moderate risk effect [odds ratio $(\mathrm{OR})=2.5$ ] and marginal risk effect $(\mathrm{OR}=3.5)$; $\mathrm{P}$ value $=0.05$; posterior power $=0.8$. The sample size of cases or controls was 470 . According to the sample sizes in the study, the posterior power was $0.790-0.809$ for the study.

The subjects fasted on the first day of hospitalization. Subsequently, a routing clinical biochemistry test was performed. Laboratory and clinical data were obtained from patient files. Hypertension was defined as blood pressure higher than 140/90 $\mathrm{mmHg}$ or being treated, diabetes mellitus was defined as fasting blood glucose above $126 \mathrm{mg} / \mathrm{dL}$ on 2 occasions or being treated (Chobanian et al., 2003; Genuth et al., 2003). Plasma glucose, urea, and Cr levels and lipid profile were measured in the fasting blood sample. Levels of total cholesterol, HDL-C, and TGs in serum were measured. LDL-C levels were calculated using the Friedewald equation (Bairaktari et al., 2000). Cr and other parameters, such as liver and kidney function, a blood clotting test, and routine blood examination, were carried out using standard methods with an auto-measuring instrument (Synchron Lx-20; Beckman-Coulter, USA) (Cockcroft and Gault, 1976). Blood samples for the DNA test were drawn and stored at $-70^{\circ} \mathrm{C}$ until use.

\section{Analysis of the KLK1 A1789G genotype}

Genomic DNA was isolated from whole blood according to standard procedures. Genotyping of KLK1 A1789G (rs5517) was performed with a TaqMan 5'-exonuclease allelic discrimination assay. The primers and probes used were as follows: forward primer: 5'-CAC AGG TGT CTT TGC CAC CTT-3'; reverse primer: 5'-CTC CCG GGT TCG TAG TCT CAT-3'; carboxyfluorescein-aminohexyl amidite-labeled probe: 5'-TTT TTC GCA CTC ATC-3'-MGB; hexachlorofluorescein-labeled probe: 5'-CTT TTT TGC ACT CAT 
C-3'-MGB. Polymerase chain reaction (PCR) was performed with 50 ng DNA in a total volume of $20 \mu \mathrm{L}$ containing $4 \mu \mathrm{L}$ reaction mix, $0.75 \mathrm{mM}$ of each primer, $0.5 \mathrm{mM}$ of each probe, and $5 \mathrm{mM} \mathrm{MgCl}_{2}$ according to manufacturer instructions for 40 cycles of a two-step PCR with denaturation at $95^{\circ} \mathrm{C}$ for $15 \mathrm{~s}$ and annealing and extension at $60^{\circ} \mathrm{C}$ for 1 min. Genotype data were read blind to the case-control status. Ambiguous genotype data were excluded from the analysis.

\section{Statistical analysis}

Continuous variables are reported as means \pm standard deviation and categorical data as percentages. Continuous variables with normal distribution were compared using the Student $t$-test, those without normal distribution were compared using the Mann-Whitney U-test, and categorical data were compared with the chi-square test. Hardy-Weinberg equilibrium (HWE) for genotype distribution was tested using the chi-square goodness-of-fit test. Variables found to have significant differences in the univariate analysis $(\mathrm{P}<0.05)$ were evaluated for binary and multinomial logistic regression. To search the association between the $K L K 1$ gene polymorphism and CAS, we adjusted the variables that had significant association with CAS. The relative ratios of CAS were estimated as ORs with associated $95 \%$ confidence intervals $(95 \% \mathrm{CIs})$. The two-sided $\mathrm{P}$ value of $<0.05$ was considered to be statistically significant. All statistical analyses were performed using SPSS 15.0 (SPSS Co., Chicago, IL, USA).

\section{RESULTS}

\section{Characteristics of case and control groups}

The characteristics of the 2 groups are shown in Table 1. The univariate analysis indicated that, in addition to age, body mass index, smoking, alcohol consumption, hypertension, diabetes, TGs, Cr, gamma-glutamyl transpeptidase, alkaline phosphatase, blood urea nitrogen, and glucose also showed significant differences with the KLK1 A1789G genotype between the 2 groups. Significant differences were absent between the 2 groups for the other factors.

\section{Polymorphism of $K L K 1$ A1789G and CAS}

The frequency distribution of the KLK1 A1789G genotype in the control group was in HWE. As shown in Table 1, the frequencies of the AA, AG, and GG (KLK1 A1789G) genotypes were $11.4,50.2$, and $38.4 \%$, respectively, in the case group and $21.2,47.7$, and $31.1 \%$, respectively, in the control group. A significant difference was found between the 2 groups (see Table 1).

A binary logistic regression model using the method of forward Wald that included the independent variables listed in Table 1 revealed the significant variables shown in Table 2. The variables of hypertension/diabetes, smoking, TGs, Cr, and the AG and GG genotypes of $K L K 1 \mathrm{~A} 1789 \mathrm{G}$ were significantly associated with increased risk of CAS. Therefore, in the next analysis of the combination of the KLK1 A1789G genotype and smoking related to CAS, the above variables as potential confounders were adjusted. 
Table 1. Comparison of clinical information between patients with coronary artery stenosis (CAS) and controls.

\begin{tabular}{|c|c|c|c|c|}
\hline Group variables & Control $(\mathrm{N}=482)$ & CAS $(\mathrm{N}=458)$ & $\chi^{2} / t$ & $\mathrm{P}$ \\
\hline Gender (male/female, \%) & $64.3 / 35.7$ & $69.9 / 30.1$ & 3.277 & 0.070 \\
\hline Age (means $\pm S$, years) & $59.17 \pm 7.81$ & $61.72 \pm 10.37$ & -4.249 & $<0.001$ \\
\hline Smoking (Yes/No, \%) & $11.2 / 88.8$ & $26.6 / 73.4$ & 36.762 & $<0.001$ \\
\hline Drinking (Yes/No, \%) & $7.9 / 92.1$ & $13.5 / 86.5$ & 7.895 & 0.005 \\
\hline Hypertention (Yes/No, \%) & $2.9 / 97.1$ & $21.0 / 79.0$ & 74.100 & $<0.001$ \\
\hline Diabetes (Yes/No, \%) & $1.2 / 98.8$ & $14.0 / 86.0$ & 55.209 & $<0.001$ \\
\hline BMI & $26.07 \pm 3.51$ & $25.84 \pm 3.40$ & 0.918 & 0.359 \\
\hline $\mathrm{TC}(\mathrm{mM})$ & $4.80 \pm 1.21$ & $4.79 \pm 1.20$ & 1.206 & 0.920 \\
\hline TGs (mM) & $1.35 \pm 0.79$ & $1.77 \pm 1.29$ & -5.872 & $<0.001$ \\
\hline $\operatorname{HDL}-\mathrm{C}(\mathrm{mM})$ & $1.35 \pm 0.58$ & $1.30 \pm 1.12$ & 0.894 & 0.371 \\
\hline LDL-C (mM) & $3.19 \pm 0.94$ & $3.09 \pm 1.10$ & 1.373 & 0.170 \\
\hline $\mathrm{A} / \mathrm{G}$ & $1.43 \pm 0.25$ & $1.46 \pm 0.25$ & -1.200 & 0.230 \\
\hline GGT (U/L) & $38.08 \pm 45.938479$ & $32.70 \pm 24.60$ & 2.188 & 0.029 \\
\hline $\mathrm{AKP}(\mathrm{U} / \mathrm{L})$ & $77.10 \pm 27.5401$ & $73.16 \pm 21.83$ & 2.744 & 0.006 \\
\hline GLU (g/L) & $5.63 \pm 1.20$ & $6.18 \pm 3.84$ & -2.875 & 0.004 \\
\hline BUN $(\mu \mathrm{M})$ & $7.39 \pm 26.85$ & $5.14 \pm 1.87$ & 1.771 & 0.077 \\
\hline $\mathrm{UA}(\mu \mathrm{M})$ & $260.41 \pm 91.94$ & $314.23 \pm 89.63$ & 0.59 & 0.631 \\
\hline $\mathrm{Cr}(\mu \mathrm{M})$ & $69.52 \pm 30.2954$ & $79.01 \pm 17.37$ & -5.801 & $<0.001$ \\
\hline $\operatorname{AST}(\mathrm{U} / \mathrm{L})$ & $30.05 \pm 27.122$ & $36.72 \pm 45.72$ & -2.650 & $<0.001$ \\
\hline PT & $13.87 \pm 12.68$ & $12.85 \pm 1.24$ & 1.662 & 0.097 \\
\hline PT-R & $0.97 \pm 0.12$ & $0.97 \pm 0.13$ & -0.956 & 0.339 \\
\hline INR & $0.97 \pm 0.12$ & $1.15 \pm 2.21$ & -1.659 & 0.097 \\
\hline FIB $(g / L)$ & $3.83 \pm 1.10$ & $3.77 \pm 1.10$ & 0.779 & 0.436 \\
\hline \multicolumn{5}{|l|}{ KLK1 A1789G genotype } \\
\hline AA & $102(21.2 \%)$ & $52(11.4 \%)$ & 17.706 & $<0.001$ \\
\hline $\mathrm{AG}$ & $230(47.7 \%)$ & $230(50.2 \%)$ & & \\
\hline GG & $150(31.1 \%)$ & $176(38.4 \%)$ & & \\
\hline
\end{tabular}

$\chi^{2}=$ chi-square test; $t=2$ independent sample Student $t$-test for quantitative variables. BMI $=$ body mass index; TC $=$ total cholesterol; TGs = plasma triglycerides; HDL-C = high-density lipoprotein cholesterol; LDL-C = low-density lipoprotein cholesterol; $\mathrm{A} / \mathrm{G}=$ albumin/globulin; $\mathrm{GGT}=$ gamma-glutamyltranspeptidase; $\mathrm{AKP}=$ alkaline phosphatase; $\mathrm{GLU}=$ glucose; BUN = blood urea nitrogen; $\mathrm{Cr}=$ creatinine; $\mathrm{AST}=$ aspartate aminotransferase; $\mathrm{PT}=$ prothrombin; $\mathrm{UA}=$ uric acid; PT-R = prothrombin-ratio; INR = international normalized ratio for clotting time; FIB = fibrinogen.

Table 2. Association between AG/GG genotypes of the $K L K 1$ A1798G gene and coronary artery stenosis in a logistic regression model.

\begin{tabular}{lccccccc}
\hline Variables $^{\mathrm{a}}$ & $\mathrm{B}$ & $\mathrm{SE}$ & Wald & d.f. & $\mathrm{P}$ & OR & $95.0 \% \mathrm{CI}$ \\
\hline Hypertension & 1.894 & 0.329 & 33.139 & 1 & 0.000 & 6.644 & $3.487-12.661$ \\
Diabetes & 1.731 & 0.508 & 11.590 & 1 & 0.001 & 5.644 & $2.084-15.284$ \\
Smoking & 0.805 & 0.233 & 11.917 & 1 & 0.001 & 2.237 & $1.416-3.534$ \\
Triglyceride & 0.507 & 0.113 & 20.154 & 1 & 0.000 & 1.661 & $1.331-2.072$ \\
Creatinine & 0.006 & 0.003 & 3.733 & 1 & 0.053 & 1.006 & $1.000-1.013$ \\
KLKI genotype & & & 9.867 & 2 & 0.007 & & \\
$\quad$ AG & 0.846 & 0.288 & 8.649 & 1 & 0.003 & 2.330 & $1.326-4.093$ \\
GG & 0.890 & 0.299 & 8.893 & 1 & 0.003 & 2.436 & $1.357-4.373$ \\
Constant + & -3.012 & 0.389 & 60.050 & 1 & 0.000 & 0.049 & \\
\hline
\end{tabular}

aSignificant variables are included in the model. (+) Constant in the binary logistic model. $\mathrm{B}=$ regression coefficient; $\mathrm{SE}=$ standard error; d.f. = degrees of freedom; $\mathrm{OR}=$ odds ratio; $95 \% \mathrm{CI}=95 \%$ confidence interval.

\section{Combination analysis of the KLK1 A1789G genotype and smoking}

Table 3 shows data regarding the effect of the combination of the KLK1 gene polymorphism and smoking on risk in the 2 groups. When we used nonsmoking with the KLK1 AA genotype as the baseline for comparison, the AG/GG genotype combined with smoking showed a remarkable increase in the risk for CAS $(\mathrm{OR}=4.53 ; 95 \% \mathrm{CI}=2.52-8.35, \mathrm{P}<0.0001)$ after adjusting for hypertension, diabetes, TGs, and $\mathrm{Cr}$, with which smoking and the KLK1 GG 
genotype greatly increased the risk $(\mathrm{OR}=4.51 ; 95 \% \mathrm{CI}=2.13-9.56, \mathrm{P}<0.0001)$. These results indicate a significant interaction between the KLK1 A1789G genotype and smoking.

Table 3. Interaction between the $K L K 1 \mathrm{~A} 1798 \mathrm{G}$ polymorphism and smoking in the risk of coronary artery stenosis.

\begin{tabular}{llccccc}
\hline Smoking & KLKl genotype & Controls [N (\%)] & Cases [N (\%)] & \multicolumn{2}{c}{ Adjusted $^{\mathrm{a}}$} & P \\
\cline { 5 - 6 } & & & & OR & $95 \%$ CI & \\
\hline No & AA & $90(18.7)$ & $34(7.4)$ & 1.00 & & \\
No & AG & $206(42.7)$ & $174(38.0)$ & 1.98 & $1.20-3.27$ & 0.007 \\
No & GG & $132(27.4)$ & $128(27.9)$ & 2.18 & $1.29-3.87$ & 0.004 \\
No & AG/GG & $338(70.1)$ & $302(67.8)$ & 2.20 & $1.38-3.50$ & 0.000 \\
Yes & AA & $12(2.5)$ & $18(3.9)$ & 2.49 & $0.91-6.79$ & 0.075 \\
Yes & AG & $24(5.0)$ & $56(12.2)$ & 3.70 & $1.83-7.50$ & 0.000 \\
Yes & GG & $18(3.7)$ & $48(10.5)$ & 4.51 & $2.13-9.56$ & 0.000 \\
Yes & AG/GG & $42(8.7)$ & $104(22.7)$ & 4.53 & $2.52-8.35$ & 0.000 \\
\hline
\end{tabular}

${ }^{a}$ Adjusted variables for triglycerides, creatinine, hypertension, and diabetes. OR $=$ odds ratio; $95 \% \mathrm{CI}=95 \%$ confidence interval.

\section{Combination analysis of the KLK1 A1789G genotype and smoking in CAS subjects with high and low stenosis scores}

In this study, CAS of $<25,26-50,51-75$, and $>75 \%$ in the left main coronary artery were $78.7,13.3,3.8$, and $4.3 \% ; 16.6,28.4,11.4$, and $43.6 \%$ in the left anterior descending branch of the left main coronary artery; $38.0,15.2,12.8$, and $34.1 \%$ in the circumflex branch of the left main coronary artery; and $30.8,20.4,14.2$, and $34.6 \%$ in the right coronary artery, respectively.

Additionally, after the evaluation of coronary angiograms, patients were also classified into a high stenosis group (stenosis score $\geq 9$ ) or a low stenosis group. Multinomial binary logistic regression analysis was performed to evaluate the effect of the combination of the $K L K 1$ genotype and smoking on risk of CAS.

Table 4 shows data for the combination of the KLK1 gene polymorphism and smok-

\begin{tabular}{|c|c|c|c|c|c|c|}
\hline \multirow[t]{2}{*}{ Smoking } & \multirow[t]{2}{*}{$K L K 1$ genotype } & \multirow[t]{2}{*}{ Controls [N (\%)] } & \multirow[t]{2}{*}{ Cases [N (\%)] } & \multicolumn{2}{|c|}{ Adjusted $^{a}$} & \multirow[t]{2}{*}{$\mathrm{P}$} \\
\hline & & & & OR & $95 \% \mathrm{CI}$ & \\
\hline \multicolumn{7}{|c|}{ Stenosis score of CAS $<9$} \\
\hline No & AA & $90(18.7)$ & $14(6.0)$ & 1.00 & & \\
\hline No & $\mathrm{AG}$ & $206(42.7)$ & $96(41.0)$ & 2.51 & $1.32-4.77$ & 0.005 \\
\hline No & GG & $132(27.4)$ & 64 (27.4) & 2.45 & $1.25-4.81$ & 0.009 \\
\hline No & $\mathrm{AG} / \mathrm{GG}$ & $338(70.1)$ & $160(68.4)$ & 2.49 & $1.34-4.63$ & 0.004 \\
\hline Yes & AA & $12(2.5)$ & $10(4.3)$ & 2.07 & $0.79-9.24$ & 0.113 \\
\hline Yes & AG & $24(5.0)$ & $24(10.3)$ & 4.56 & $1.94-10.72$ & $<0.001$ \\
\hline Yes & GG & $18(3.7)$ & $26(11.1)$ & 6.53 & $2.74-15.57$ & $<0.001$ \\
\hline Yes & $\mathrm{AG} / \mathrm{GG}$ & $42(8.7)$ & $50(21.4)$ & 5.42 & $2.60-11.31$ & $<0.001$ \\
\hline \multicolumn{7}{|c|}{ Stenosis score of $\mathrm{CAS} \geq 9$} \\
\hline No & AA & $90(18.7)$ & $20(8.9)$ & 1.00 & & \\
\hline No & $\mathrm{AG}$ & $206(42.7)$ & $78(34.8)$ & 1.59 & $0.85-2.96$ & 0.142 \\
\hline No & GG & $132(27.4)$ & 64 (28.6) & 2.38 & $1.26-4.48$ & 0.007 \\
\hline No & $\mathrm{AG} / \mathrm{GG}$ & $338(70.1)$ & $142(63.4)$ & 1.90 & $1.06-3.42$ & 0.031 \\
\hline Yes & AA & $12(2.5)$ & $8(3.6)$ & 3.86 & $1.56-9.53$ & 0.044 \\
\hline Yes & $\mathrm{AG}$ & $24(5.0)$ & $32(14.3)$ & 4.16 & $1.82-9.50$ & 0.001 \\
\hline Yes & GG & $18(3.7)$ & $22(9.8)$ & 3.86 & $1.56-9.53$ & 0.003 \\
\hline Yes & $\mathrm{AG} / \mathrm{GG}$ & $42(8.7)$ & $54(24.1)$ & 4.07 & $1.97-8.38$ & $<0.001$ \\
\hline
\end{tabular}

${ }^{\mathrm{a}}$ Adjusted variables for triglycerides, creatinine, hypertension, and diabetes. OR $=$ odds ratio; $95 \% \mathrm{CI}=95 \%$ confidence interval. 
ing on the risk of CAS in the high and low stenosis groups. When we used nonsmoking with the KLK1 AA genotype as the baseline for comparison, the AG/GG genotype combined with smoking showed a remarkable increase in the risk of CAS in the low stenosis group $(\mathrm{OR}=5.42$; $95 \% \mathrm{CI}=2.60-11.31, \mathrm{P}<0.001)$ and the high stenosis group $(\mathrm{OR}=4.07 ; 95 \% \mathrm{CI}=1.97-8.38, \mathrm{P}$ $<0.001)$ after adjusting for hypertension, diabetes, TGs, and $\mathrm{Cr}$. The result indicates a significant interaction between the KLK1 A1789G genotype and smoking in the early development stage of CAS.

\section{DISCUSSION}

Our results suggest that the GG genotype of the KLK1 A1789G (rs5517) gene is associated with CAS in the Chinese Han population. Previous studies have shown that KLK1 is associated with cardiovascular disorders (Sharma and Kesavarao, 1996; Sharma et al., 1998, 1999). KLK1 is a member of kallikrein family of serine proteases and belongs to a subgroup of serine proteinases. It processes low molecular weight kininogen substrates to release vasoactive kinin peptides. Intact kinins bind to bradykinin B2 receptors, whereas kinin metabolites produced by enzymes such as kinases I and II and neutral endopeptidase bind to bradykinin B1 receptors.

In vivo pretreatment of littermate wild-type mice with a B2 kinin receptor antagonist reproduced the effects of tissue kallikrein deficiency. However, like wild-type mice, B2 receptor-deficient mice [B2 (-/-)] unexpectedly responded to ischemic preconditioning or angiotensin-converting enzyme inhibitor (Griol-Charhbili et al., 2005). The cardioprotective effects of angiotensin-converting enzyme inhibitors on ischemia-reperfusion injury are the result of the protection of endogenous kinins from degradation rather than inhibition of angiotensin II formation (Liu et al., 1996). The binding of kinins to their respective receptors activates signaling pathways such as nitric oxide-cyclic guanosine monophosphate and prostacyclin-cyclic adenosine monophosphate, which trigger a broad spectrum of biological effects including endothelial function in arteries, vasodilatation, smooth muscle contraction and relaxation, inflammation, and pain (Griol-Charhbili et al., 2005). Therefore, the KLK1 A1789G polymorphism is likely to be directly related to the change function of the B2 kinin receptor and carcinoembryonic antigen inhibitor.

Several KLK1 gene polymorphisms have been identified, and a few genetic variants may influence urinary kallikrein activity (Chao and Chao, 2005). Some investigators have found that the $K L K 1$ gene polymorphism is associated with blood pressure regulation in spontaneously hypertensive rats (Lu et al., 2007). A study involving 57 Utah pedigrees indicated that a dominant allele expressed as high urinary kallikrein excretion was associated with a decreased risk of essential hypertension (Zhao et al., 2007). The A1789G (rs5517) polymorphism of the KLK1 gene exon 4 is a single-nucleotide substitution that causes the amino acid substitution Lys $\rightarrow$ Glu. Plasma Cr levels in patients with hypertension carrying the mutant allele $\mathrm{G}$ (AG or GG genotype) are significantly higher than those in patients carrying allele A (AA genotype) (Pravenec et al., 1991).

Of particular interest, serum $\mathrm{Cr}$ was shown to be related to CAS. Cr has frequently been used as a preferred marker of renal function in practice (Zhao et al., 2007). Novel risk factors associated with renal dysfunction may play a role in adverse cardiovascular outcomes in patients with allele A and are being currently investigated. Inflammation, altered calcium- 
phosphate homeostasis, increased oxidative stress, and elevated homocysteine and uric acid levels collaborate and, hence, accelerate atherosclerosis and endothelial dysfunction (Shlipak, 2003). A study has reported that even mild elevations of serum $\mathrm{Cr}$ might be considered an independent sign of more extensive atherosclerotic involvement of coronary arteries (National Kidney Foundation, 2002). However, whether the link between Cr and CAS is a causal relationship or a reaction to the disease remains unclear.

To our knowledge, the present study is the first to report that allele $\mathrm{G}$ is associated with an increased risk of CAS compared with that associated with allele A. In one animal study, bradykinin coronary outflow, left ventricular performance, and left ventricular dimensions of transgenic rats harboring $K L K 1$ were investigated under basal and ischemic conditions. Compared to controls, rats with $K L K 1$ displayed a basal bradykinin outflow that was increased 3.5 -fold. Ischemia induced an increase of bradykinin coronary outflow in controls but did not induce further increase in rats in the $K L K 1$ group. However, despite similar unchanged infarction sizes, left ventricular function and remodeling improved in the $K L K 1$ group after myocardial infarction (Koch et al., 2006). These results provide reasonable evidence of a relationship between the KLK1 A1789G gene polymorphism and increased risk of CAS. A larger sample size is needed to confirm a significant association to CAS.

The major finding of the current study is that allele $\mathrm{G}$ of the $K L K 1$ gene increases the risk of CAS, has an interactive effect with smoking, and is important in the prevention of CAS after adjusting for known risk factors such as hypertension, diabetes, and dyslipidemia. Hence, smoking is a risk factor for CAS (Kones, 2010). Köz et al. (2009) studied 53 patients younger than 45 years with severe CAS treated with angiography (group A) and 60 age-matched patients with normal or non-critical stenosis treated with coronary angiography (group B). The authors found that smoking was an independent predictor of severe CAD in young patients according to logistic regression analysis, with an $\mathrm{OR}$ of $3.7(95 \% \mathrm{CI}=0.572-8.763 ; \mathrm{P}=0.002)$. Wong et al. (2005) associated current cigarette smoking $(\mathrm{OR}=3.08 ; 95 \% \mathrm{CI}=1.47-6.47)$ with cardiovascular diseases using data pooled from the Atherosclerosis Risk in Communities Study $(\mathrm{N}=12,642$; mean age, 60 years) and the Cardiovascular Health Study ( $\mathrm{N}=2824$; mean age, 79 years). Recent research has indicated that cigarette smoking is related to abnormal peripheral and coronary vascular vasomotion (Morita et al., 2006). The results of another report showed that coronary vascular endothelial dysfunction was caused by chronic smoking in smokers without epicardial CAS and that coronary endothelial dysfunction may be reversible within 1 month after smoking cessation in healthy young smokers (Wilson et al., 1998).

In the present study, smoking was associated with CAS $(\mathrm{OR}=2.715 ; 95 \% \mathrm{CI}=1.703$ 4.307). However, an individual smoker with allele $\mathrm{G}$ in the $K L K 1 \mathrm{~A} 1789 \mathrm{G}$ gene has a prominently increased risk $(\mathrm{OR}=5.42 ; 95 \% \mathrm{CI}=2.60-11.31$ in the low-score stenosis group and $\mathrm{OR}=4.07 ; 95 \% \mathrm{CI}=1.97-8.38$ in the high-score stenosis group). This finding indicates that smoking with $K L K 1 \mathrm{GG}$ was significantly associated with an increased risk of CAS compared with nonsmoking with $K L K 1 \mathrm{AA}$, especially in the early development stage of the disease.

Hypertension, diabetes, lipids, age, smoking, and alcohol consumption clearly increase the risk of CAD (Berenson et al., 1998). TG and LDL-C in subjects with CAD are higher than those in subjects without CAD (da Luz et al., 2005). One study has shown that the TG/HDL-C ratio is significantly correlated to coronary atherosclerosis (Bampi et al., 2009). Diabetic subjects have a greater atherosclerotic plaque burden and more severe coronary atherosclerosis than those without diabetes. Most obstructive lesions are caused by mixed plaques 
in diabetic and nondiabetic patients (Gao et al., 2011). In the present study, we found that hypertension, diabetes, TGs, and smoking were associated with CAS, and the results were consistent with those of the previous report (Berenson et al., 1998).

\section{Study limitations}

Subjects with CAS in this study were diagnosed using ECG, cardiac ultrasound, and coronary angiography examinations. The controls were diagnosed with ECG and cardiac ultrasound examination, but they did not undergo coronary angiography examination. We could expect a very low prevalence of asymptomatic CAS in the control group, so the results of our study are reliable. However, CAS was not fully ruled out in the controls, because the subjects in the control group did not undergo examinations such as scintigraphy, exercise stress tests, and coronary computed tomography. This characteristic is a limitation in the study.

\section{Summary}

We found that the GG genotype of the KLK1 A1789G gene is associated with CAS, and smoking with $K L K 1 \mathrm{GG}$ is significantly associated with an increased risk of CAS compared with the risk associated with nonsmoking and $K L K 1 \mathrm{AA}$, especially in the early development stage of the disease. This result is extremely important in the development of prevention strategies for CAS.

\section{Conflict of interests}

The authors declare that they have no competing interests.

\section{ACKNOWLEDGMENTS}

Research supported by the Natural Science Foundation of Shandong Province (\#Y2007C042) and the Doctor Fund Projects of Shandong Province (\#9915, \#BS2009YY049 and \#2010BSE27061).

\section{REFERENCES}

Bairaktari E, Hatzidimou K, Tzallas C, Vini M, et al. (2000). Estimation of LDL cholesterol based on the Friedewald formula and on apo B levels. Clin. Biochem. 33: 549-555.

Bampi AB, Rochitte CE, Favarato D, Lemos PA, et al. (2009). Comparison of non-invasive methods for the detection of coronary atherosclerosis. Clinics 64: 675-682.

Berenson GS, Srinivasan SR, Bao W, Newman WP III, et al. (1998). Association between multiple cardiovascular risk factors and atherosclerosis in children and young adults. The Bogalusa Heart Study. N. Engl. J. Med. 338: 1650-1656.

Berry TD, Hasstedt SJ, Hunt SC, Wu LL, et al. (1989). A gene for high urinary kallikrein may protect against hypertension in Utah kindreds. Hypertension 13: 3-8.

Chao J and Chao L (2005). Kallikrein-kinin in stroke, cardiovascular and renal disease. Exp. Physiol. 90: 291-298.

Chobanian AV, Bakris GL, Black HR, Cushman WC, et al. (2003). Seventh report of the Joint National Committee on Prevention, Detection, Evaluation, and Treatment of High Blood Pressure. Hypertension 42: 1206-1252.

Cockcroft DW and Gault MH (1976). Prediction of creatinine clearance from serum creatinine. Nephron 16: 31-41.

da Luz PL, Cesena FH, Favarato D and Cerqueira ES (2005). Comparison of serum lipid values in patients with coronary artery disease at $<50,50$ to 59, 60 to 69, and $>70$ years of age. Am. J. Cardiol. 96: 1640-1643. 
Gao Y, Lu B, Sun ML, Hou ZH, et al. (2011). Comparison of atherosclerotic plaque by computed tomography angiography in patients with and without diabetes mellitus and with known or suspected coronary artery disease. Am. J. Cardiol. 108: 809-813.

Genuth S, Alberti KG, Bennett P, Buse J, et al. (2003). Follow-up report on the diagnosis of diabetes mellitus. Diabetes Care 26: 3160-3167.

Griol-Charhbili V, Messadi-Laribi E, Bascands JL, Heudes D, et al. (2005). Role of tissue kallikrein in the cardioprotective effects of ischemic and pharmacological preconditioning in myocardial ischemia. FASEB J. 19: 1172-1174.

Hong ZY, Zhang XQ, Huang G, Ling DQ, et al. (2005). Effects of human tissue kallikrein gene A1789G polymorphism on plasma creatinine levels in patients with essential hypertension. Chin. J. Clin. Pharmacol. Therapeut. 10: 637-641.

Koch M, Spillmann F, Dendorfer A, Westermann D, et al. (2006). Cardiac function and remodeling is attenuated in transgenic rats expressing the human kallikrein-1 gene after myocardial infarction. Eur. J. Pharmacol. 550: 143-148.

Kones R (2010). Recent advances in the management of chronic stable angina I: approach to the patient, diagnosis, pathophysiology, risk stratification, and gender disparities. Vasc. Health Risk Manag. 6: 635-656.

Köz C, Celebi H, Yokusoglu M, Baysan O, et al. (2009). The relation between coronary lesion distribution and risk factors in young adults. Anadolu Kardiyol. Derg. 9: 91-95.

Liu YH, Yang XP, Sharov VG, Sigmon DH, et al. (1996). Paracrine systems in the cardioprotective effect of angiotensinconverting enzyme inhibitors on myocardial ischemia/reperfusion injury in rats. Hypertension 27: 7-13.

Lu X, Zhao W, Huang J, Li H, et al. (2007). Common variation in KLKB1 and essential hypertension risk: tagging-SNP haplotype analysis in a case-control study. Hum. Genet. 121: 327-335.

Morita K, Tsukamoto T, Naya M, Noriyasu K, et al. (2006). Smoking cessation normalizes coronary endothelial vasomotor response assessed with 15O-water and PET in healthy young smokers. J. Nucl. Med. 47: 1914-1920.

National Kidney Foundation (2002). K/DOQI clinical practice guidelines for chronic kidney disease: evaluation, classification, and stratification. Am. J. Kidney Dis. 39: S261-S266.

Pravenec M, Kren V, Kunes J, Scicli AG, et al. (1991). Cosegregation of blood pressure with a kallikrein gene family polymorphism. Hypertension 17: 242-246.

Sharma JN and Kesavarao U (1996). Cardiac kallikrein in hypertensive and normotensive rats with and without diabetes. Immunopharmacology 33: 341-343.

Sharma JN, Uma K and Yusof AP (1998). Left ventricular hypertrophy and its relation to the cardiac kinin-forming system in hypertensive and diabetic rats. Int. J. Cardiol. 63: 229-235.

Sharma JN, Kesavarao U and Yusof AP (1999). Altered cardiac tissue and plasma kininogen levels in hypertensive and diabetic rats. Immunopharmacology 43: 129-132.

Shlipak MG (2003). Pharmacotherapy for heart failure in patients with renal insufficiency. Ann. Intern. Med. 138: 917-924.

Slim R, Torremocha F, Moreau T, Pizard A, et al. (2002). Loss-of-function polymorphism of the human kallikrein gene with reduced urinary kallikrein activity. J. Am. Soc. Nephrol. 13: 968-976.

Wilson PW, D'Agostino RB, Levy D, Belanger AM, et al. (1998). Prediction of coronary heart disease using risk factor categories. Circulation 97: 1837-1847.

Wong TY, Larsen EK, Klein R, Mitchell P, et al. (2005). Cardiovascular risk factors for retinal vein occlusion and arteriolar emboli: the Atherosclerosis Risk in Communities \& Cardiovascular Health studies. Ophthalmology 112: 540-547.

Yeolekar ME and Jadhav L (1993). Concept of cardiovascular risk and its clinical application. South Asian J. Prev. Cardiol. 5: 58-78.

Yu HZ, Xie LD, Zhu PL, Xu CS, et al. (2010). Effects of human tissue kallikrein 1 gene delivery on carotid artery neointima formation after balloon angioplasty in spontaneously hypertensive rats. Zhonghua Xin. Xue Guan. Bing. Za Zhi 38: 67-71.

Zhao W, Wang L, Lu X, Yang W, et al. (2007). A coding polymorphism of the kallikrein 1 gene is associated with essential hypertension: a tagging SNP-based association study in a Chinese Han population. J. Hypertens. 25: 1821-1827. 